\title{
Reduction in the ionospheric error for a single-frequency GPS timing solution using tomography
}

\author{
Julian A.R. Rose, Damien J. Allain and Cathryn N. Mitchell \\ Invert Centre for Imaging Science, Department of Electronic and Electrical Engineering, \\ University of Bath, UK
}

\begin{abstract}
Single-frequency Global Positioning System (GPS) receivers do not accurately compensate for the ionospheric delay imposed upon a GPS signal. They rely upon models to compensate for the ionosphere. This delay compensation can be improved by measuring it directly with a dual-frequency receiver, or by monitoring the ionosphere using real-time maps. This investigation uses a 4D tomographic algorithm, Multi Instrument Data Analysis System (MIDAS), to correct for the ionospheric delay and compares the results to existing single and dualfrequency techniques. Maps of the ionospheric electron density, across Europe, are produced by using data collected from a fixed network of dual-frequency GPS receivers. Single-frequency pseudorange observations are corrected by using the maps to find the excess propagation delay on the GPS L1 signals. Days during the solar maximum year 2002 and the October 2003 storm have been chosen to display results when the ionospheric delays are large and variable. Results that improve upon the use of existing ionospheric models are achieved by applying MIDAS to fixed and mobile single-frequency GPS timing solutions. The approach offers the potential for corrections to be broadcast over a local region, or provided via the internet and allows timing accuracies to within $10 \mathrm{~ns}$ to be achieved.
\end{abstract}

Key words GPS - ionosphere - tomography - timing - single-frequency

\section{Introduction}

The Global Positioning System (GPS) allows a user to solve for their position and time virtually anywhere in the world. GPS receivers use Time Difference of Arrival (TDOA) techniques to measure the time of signal propagation between each satellite in view and the receiver, together with the knowledge of the satel-

Mailing address: Mr. Julian A.R. Rose, Invert Centre for Imaging Science, Department of Electronic and Electrical Engineering, University of Bath, Bath, BA2 7AY, UK; e-mail: J.A.R.Rose@bath.ac.uk lite positions, to compute a solution. When the GPS signals propagate through the ionosphere they are both slowed and refracted which directly affects the accuracy of the solution. This effect is proportional to the line integral of the ionospheric electron density, the Total Electron Content (TEC), which is dependent upon several factors: season, time of day, solar activity and latitude, to name but a few. Hence, an unknown, highly variable propagation delay is imposed upon the signals (Davies, 1990).

Civilians commonly use GPS receivers for satellite navigation. They operate on a singlefrequency, the $L 1$ channel and are now compact and affordable. However, their accuracy is hindered by the ionospheric delay, which is the cause of the largest error in a single-frequency solution (Langley, 1997). This delay is largely removed by dual-frequency GPS receivers, 
which hence provide more accurate solutions and are used by the scientific community towards atmospheric monitoring for example. However, dual-frequency receivers are more expensive than standard single-frequency receivers and in extreme ionospheric conditions, are susceptible to losses of lock on the $L 2$ signal, which corrupts the GPS solution.

There are ionospheric models available to single-frequency users that compensate for this delay. The GPS satellites broadcast the Klobuchar model coefficients via the GPS navigation message (Klobuchar, 1987). This aims to provide at least a 50\% Root Mean Square (RMS) correction for the ionospheric time delay. Another model, known as the International Reference Ionosphere (IRI), is available (Bilitza, 2001), but not in real-time to GPS users. Geostationary satellite systems approximate the ionosphere to a thin shell and broadcast realtime ionospheric mapping information to complement the GPS solution. North America, Europe and Asia employ the Wide Area Augmentation System (WAAS), European Geostationary Navigation Overlay System (EGNOS) and Multi-functional Satellite Augmentation System (MSAS) respectively.

Single-frequency GPS receivers, coupled with the 4D tomographic algorithm, Multi Instrument Data Analysis System (MIDAS) (Mitchell and Spencer, 2003), present a cheaper alternative to dual-frequency receivers and a more accurate alternative to standard single-frequency receivers. Tomographic imaging allows for a realistic estimate of the ionosphere to be created, see for example the review by Bust and Mitchell (2008). This real-time mapping system, MIDAS, provides the ionospheric delay by producing electron density images (maps), which have been proven to provide more accurate representations of the ionosphere than $2 \mathrm{D}$ shell approximations (e.g. WAAS) which introduce mapping function errors (Meggs and Mitchell, 2006). Smith et al. (2008) state that thin shell models fail to describe the TEC of a slanted ray path because they do not contain any information regarding the vertical structure of the ionosphere. The MIDAS maps show the time evolution of plasma across the ionosphere, allowing real-time corrections to be made. A large network of GPS receivers is required over the area of interest in order to monitor and track the plasma. This is already in place within Europe through the International GNSS (Global Navigation Satellite System) Service (IGS) and the Regional Reference Frame Sub-Commission for Europe (EUREF) Permanent Network (EPN). The IGS and EPN are voluntary services and collectively consist of hundreds of fixed GNSS stations that provide high precision products.

The aim of this paper is to show that the accuracy of a single-frequency GPS timing solution can be improved by using 4D tomographic mapping to reduce the ionospheric delay. Results are presented to this effect by including comparisons between timing solutions that are obtained using different ionospheric corrections. The figures show solutions for fixed and mobile receivers with: no correction, the Klobuchar model, the IRI 2001 model, the realtime MIDAS maps and dual-frequency corrections. The latter is taken as the benchmark timing solution because it essentially removes the ionospheric delay by providing a direct means of measuring it.

It should be noted from the outset that these results represent the GPS solutions for a freerunning GPS timing device, regardless of location and show how accurately we can bound the error. This is analogous to a navigational solution that is relative to the center of the Earth, as opposed to a solution that is relative to a known, fixed and local point. In systems such as the latter, Differential GPS (DGPS) for example, common errors are cancelled out, which greatly enhances the solution accuracy. The dual-frequency results presented here should not be compared to precise time-transfer techniques, such as Two-Way Satellite Time and Frequency Transfer (TWSTFT), which can obtain $0.1 \mathrm{~ns}$ accuracy and is expensive to operate as it requires a dedicated satellite link (Ray and Senior, 2003). This is because we present instantaneous solutions for fixed and mobile GPS receivers and do not present results that use common view geostationary or GPS satellites to synchronise two clocks at different locations, nor are these results averaged over any lengthy time periods. Averaging over several days can 
improve the accuracy by smoothing-out noise in time-transfers (Lewandowski and Azoubib, 2000). Therefore, this research does not aim to compete with high precision time-transfer techniques, but instead focuses on improving the accuracy of existing single-frequency GPS timing solutions that are globally obtainable from the standard GPS constellation.

The results are in the form of a receiver clock bias; this is the offset between the GPS receiver clock and GPS Time (GPST), which is referenced to the receiver clock bias as calculated by the Centre for Orbit Determination in $\mathrm{Eu}-$ rope (CODE) (Beutler et al., 1999). From this point on, the Receiver Clock Bias shall be referred to as the RCB. The CODE, an IGS Analysis Center, uses the GPS code and phase measurements, together with atomic clock references to calculate their clock solutions (Kouba and Springer, 2001). This yields a precise timing solution at the test station and is taken as the 'truth', to give an independent and fair comparison between methods. These receiver clock products are computed daily and are freely available to download from the IGS (Dow et al., 2005). The RCB, calculated by each of the five timing solutions, are presented relative to the bias as calculated by CODE. An offset exists between these solutions and the solution provided by CODE due to differences in filtering to remove multipath.

\section{Method}

\subsection{Overview}

The method described in this section is similar to the method presented by Allain and Mitchell (2008), which involves using tomography to correct for the ionosphere and measures its impact upon GPS positioning. In contrast this paper is concerned with fixed and mobile single-frequency GPS receiver timing solutions, each of which incorporates a different ionospheric correction technique.

In order to represent different geophysical conditions both quiet and stormy days have been selected for this study from the year 2002, along with a stormy period during October 2003. The selection was based upon Kp index, which ranges from 0-9 and gives a measure of the disturbance in the Earth's magnetic field. The Kp values were obtained from the UK Solar System Data Centre (UKSSDC), see http://www.ukssdc.ac.uk/.

\subsection{IGS/EPN Stations, Map and MIDAS}

Data from dual-frequency GPS receiver sites across mainland Europe have been obtained from the IGS (Dow et al., 2005) and the EPN (Bruyninx, 2004) for this study. The data at each site is recorded at $30 \mathrm{~s}$ intervals in Receiver Independent Exchange (RINEX) format (Gurtner, 2002). Stations in close proximity to each other were not chosen because they fail to provide any additional data about the ionosphere for that region. As a result 74 stations located across Europe were used to create ionospheric maps.

Two IGS stations, based in different areas of Europe, are used as test stations. Single-frequency GPS data from these sites are used to illustrate the performance of a single-frequency GPS receiver with: no ionospheric correction, the Klobuchar ionospheric correction, the IRI 2001 correction and finally the MIDAS correction. The dual-frequency data, available from these test sites, are then included in the resulting figures to enable direct comparisons between a wide range of GPS timing solutions. The data from these test stations does not contribute to the ionospheric mapping since independent single-frequency solutions are desired. The test stations are VILL (Villafranca, Spain) and GOPE (Ondrejov, Czech Republic). Figure 1 shows the location of the test stations and those used for the imaging (or 'inversion'), by representing each site with a red and green marker respectively.

The tomographic grid is centered on Europe at $50^{\circ} \mathrm{N} 15^{\circ} \mathrm{E}$. The longitude and latitude ranges from $-44^{\circ}$ to $+44^{\circ}$ in steps of $4^{\circ}$. The altitude ranges from $100 \mathrm{~km}$ to $1500 \mathrm{~km}$ in steps of 50 $\mathrm{km}$. The MIDAS inversions use three empirical orthonormal functions, to represent the vertical basis functions and are computed from a range 


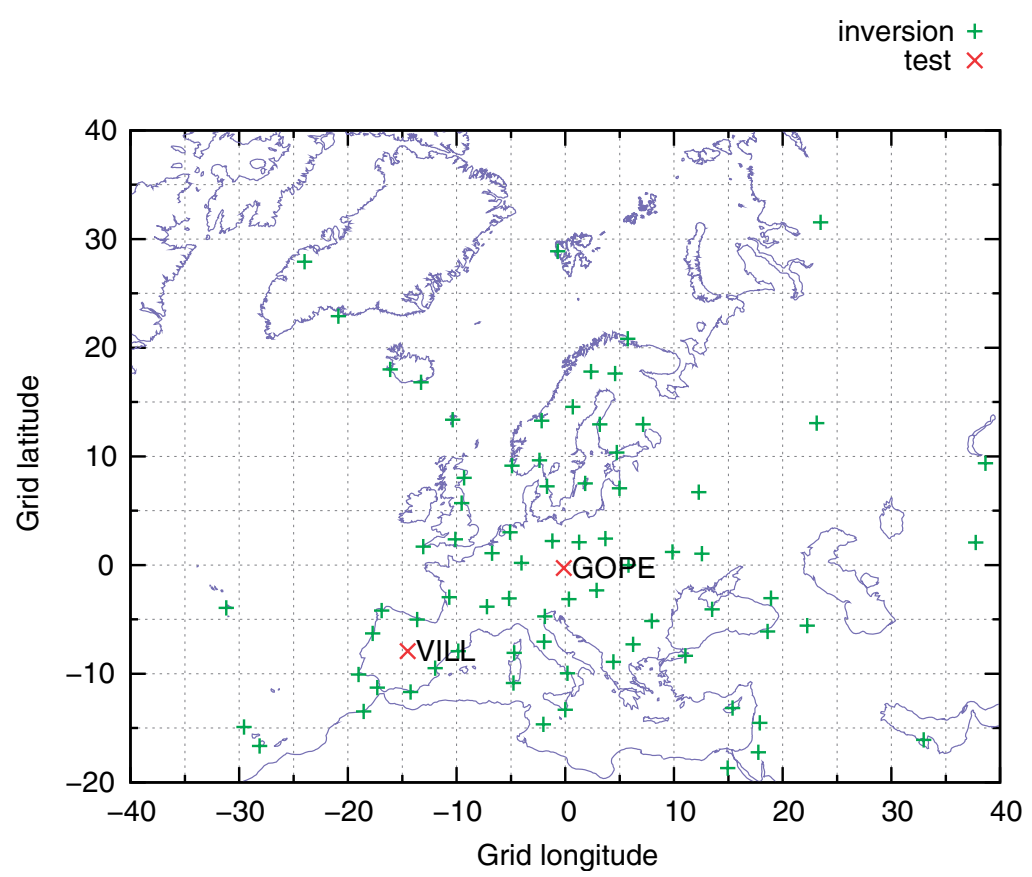

Fig. 1. Map of Europe, showing the test stations and those used for the inversion.

of Epstein functions. For a detailed description of the MIDAS tomographic algorithms please see Mitchell and Spencer (2003) and Spencer and Mitchell (2007).

\subsection{Timing solution overview}

GPS satellites are synchronised to GPST and their positions are provided by the IGS. The GPS receiver records the local Time of Arrival (TOA), or 'time of reception', of a GPS signal according to its clock. Each received navigation message incorporates the time at which the Satellite Vehicle (SV) broadcast the signal, the Time of Transmission (TOT). Thus the pseudorange between each satellite and the receiver may be computed. However, it is not that simple. Parkinson and Spilker (1996) state that the pseudorange may be defined as the difference between the satellite clock time and the receiver clock time, but it is corrupted by the receiver's clock bias which must be estimated and re- moved. Additionally, there are several biases and propagation errors which are imposed upon the GPS signal and system as a whole and so several corrections are applied to the pseudorange equation, before the ionospheric correction, that are fundamental and common to each of the GPS timing solution techniques presented in this paper.

If the GPS receiver is at a known position then the receiver clock bias, $b_{r x}$, can be obtained from a single satellite. The fundamental and common corrections may be calculated using the known position. This yields the RCB and as there are typically several satellites in view, a more accurate $b_{r x}$ value is obtained by averaging all of the values of $b_{r x}$ for all of the satellites in view at each instant in time. In contrast, if the GPS receiver is at an unknown position it must track at least four satellites, to form at least four pseudorange equations, to solve for its position coordinates $x, y, z$ and its $\mathrm{RCB}$, using a least squares technique. The fundamental and common corrections must first be estimated using an 
approximation of the receiver coordinates. More accurate pseudorange equations may then be formed and applied to the least squares technique for a second time in order to output more accurate receiver coordinates. This process is repeated to obtain a more accurate $x, y, z$ and $b_{r x}$.

The RCB represents the receiver clock's offset from actual GPS system time. Therefore, once this value has been found, the GPST at the receiver's location is known and may be converted to Universally Coordinated Time (UTC) using the specific broadcast conversion parameter. This technique is employed by each of the five GPS timing solutions, together with the common corrections and the only difference between them is the method by which the ionospheric delay is accounted for. Each solution yields the RCB at the receiver, which is then presented relative to the bias as calculated by CODE.

\subsection{Pseudorange}

The pseudorange, $P$, is the difference between the satellite clock time and receiver clock time and in the idealised error-free condition is as follows, in seconds:

$$
P=t_{r}-t_{t}
$$

where $t_{r}$ is the GPST at the receiver (time of reception) and $t_{t}$ is the GPST at the satellite (time of transmission). But it is also corrupted by the clock in the GPS receiver, the 'RCB', $b_{r x}$ :

$$
P=\left(t_{r}-t_{t}\right)+b_{r x}
$$

Realistically, there are propagation errors, relativistic effects and various other biases and noise effects and so the pseudorange, as measured by the GPS receiver, becomes (in seconds):

$$
P=\left(t_{r}-t_{t}\right)+b_{r x}+b_{s v}+T_{\text {sagn }}+T_{\text {trop }}+T_{\text {iono }}+\varepsilon
$$

where $T_{\text {sagn }}$ is the Sagnac bias, $T_{\text {trop }}$ is the tropospheric delay, $T_{\text {iono }}$ is the delay imposed by the ionosphere, $b_{s v}$ is the satellite clock bias (accounts for the satellite specific bias and rela- tivistic effects) and $\varepsilon$ represents any unmodelled errors (which are ignored). Several corrections are applied to each of the timing solutions to account for the above delays or biases, but before they can be calculated several other fundamental corrections and calculations must be performed.

\subsection{Fundamental corrections and calculations}

This section describes the fundamental corrections and calculations which are necessary in order to compute the GPS solution.

\section{- Differential Code Bias (DCB)}

The two codes, modulated onto the $L 1$ and L2 frequencies, have different electrical path lengths at the transmitter/receiver, which results in the so called Differential Code Bias (DCB), which are specific to each satellite and receiver and are provided by the CODE. The satellites show a small $P 1$ to $P 2$ bias, $\Delta_{s}^{1-2} t$, which gives a different clock bias for each frequency, $f_{l}$ and $f_{2}$. The single-frequency clock bias, $\Delta_{s}^{1} t$ is offset from the dual-frequency clock bias, $\Delta_{s} t$ (which is given in the ephemeris data) as follows:

$$
\Delta_{s}^{1} t=\Delta_{s} t+\frac{f_{1}^{2-}}{f_{1}^{-2}-f_{2}^{-2}} \Delta_{s}^{1-2} t
$$

\section{- Antenna Offsets}

The ephemeris data are calculated with respect to the satellite's centre of mass, whereas signal measurements are made with respect to the Antenna Phase Center (APC). These offsets are calculated by Schmid et al. (2007) for each satellite. The position of the antenna is found as follows:

$$
\vec{S} \simeq \frac{S^{\prime}-\Delta_{v} S}{S^{\prime}} \vec{S}^{\prime}
$$

where $\vec{S}$ is the antenna position, $S^{\prime}$ is the modulus of the satellite center of mass position vector, $\Delta_{v} S$ is the vertical component of the antenna offset and $\vec{S}^{\prime}$ is the position vector of the satellite center of mass. The horizontal satellite antenna offsets are neglected because they are extremely complicated to take into account and have an insignificant impact upon the range. 
- Satellite position, $\vec{S}$ and Time of transmission, $t_{t}$

The satellite positions are given by the ephemeris data and are presented in Earth-Centered Earth-Fixed (ECEF) frame coordinates at intervals of $15 \mathrm{~min}$ (Dow et al., 2005). The antenna coordinates are interpolated from the ephemeris (with the antenna offsets applied) at the TOT. Trigonometric interpolation, with 4 harmonics in the ECEF frame, is used as follows:

$$
\vec{S} \simeq \sum_{i=1}^{4} \vec{A}_{i} \sin i \Omega t+\vec{B}_{i} \cos i \Omega t
$$

where $\vec{S}$ is the satellite position, $i$ is the index of the harmonic, $\Omega$ is the angular velocity of the Earth, $t$ is the time and $\vec{A}_{i}$ and $\vec{B}_{i}$ are the amplitude coefficients of the harmonic.

However, $t_{t}$ is not explicitly recorded in the RINEX file and so iterative methods are used to extract it. It can be calculated provided the time of reception, $t_{r}$ (recorded in the RINEX file) and the range between the satellite and receiver $r$ are known:

$$
t_{t}=t_{r}-r / c
$$

where $c$ is the speed of light and the range can be calculated from the receiver and satellite coordinates, $R$ and $S$ respectively. But since $S$ is a function of transmission time an iterative approach is required to find it. A first approximation of the receiver coordinates, $R$, may be found using the least squares method, described later.

The rate at which the distance between a GPS satellite and a ground-based receiver changes is:

$$
|\dot{r}|<800 m s^{-1}
$$

The uncertainty on the range, $\Delta r$, and the uncertainty of the transmission time, $\Delta t_{t}$, are related as follows:

$$
\Delta r=|\dot{r}| \Delta t_{t} \text { and } \Delta t_{t}<c^{-1} \Delta r
$$

The computation converges quickly towards the solution because each iteration multiplies the uncertainty on the range by:

$$
c^{-1}|\dot{r}|<2.7 \times 10^{-6}
$$

The range, $r$, between a GPS satellite and a ground based receiver must lie between $20.2 \times$ $10^{6} \mathrm{~m}$ and $\sim 26.6 \times 10^{6} \mathrm{~m}$, ignoring any delays, as follows (in metres):

$$
r=(23.4 \pm 3.2) \times 10^{6}
$$

The aim is to achieve an uncertainty of $\Delta r$ $<0.1 \mathrm{~m}$, which leads to the number of iterations required, 2, as follows:

$$
\frac{\ln \left(0.1 / 3.2 \times 10^{6}\right)}{\ln (|\dot{r}| / c)} \leq 2
$$

Results with an uncertainty of approximately $0.04 \mathrm{~m} \mathrm{RMS}$ and a maximum of $0.15 \mathrm{~m}$ were achieved.

\section{- Satellite velocity, $\overrightarrow{\dot{S}}$}

Satellite velocities are found by multiplying the coefficients, found from the position interpolation, with the derivatives of the trigonometric functions, also used in the interpolation of the satellite positions:

$$
\overrightarrow{\dot{S}} \simeq \sum_{i=1}^{4} i \Omega\left(-\vec{A}_{i} \sin i \Omega t+\vec{B}_{i} \cos i \Omega t\right)
$$

where $\overrightarrow{\dot{S}}$ is the satellite velocity and the other terms are as described previously under the heading 'satellite position'. The precise satellite positions and velocities are hence used to calculate the relativistic clock bias of the satellites and the Sagnac bias.

\subsection{Least squares technique}

A least squares technique is used to solve for the position $(x, y, z)$ and clock bias $\left(b_{r x}\right)$ of a GPS receiver. The method shown below originates from Parkinson and Spilker (1996) and is used by Allain and Mitchell (2008).

If each satellite is given a number, $n$ and $r$ is the range between satellite and receiver, then 
for $\mathrm{n} \geq 4$ satellites, taking a receiver of position $R$ and satellite of position $S_{i}$, with $1 \leq i \leq \mathrm{n}$, then the pseudorange between a specific satellite, $i$, and receiver, ignoring errors, is as follows (in metres):

$$
P_{i}=c b_{r x}+r_{i}
$$

where $c$ is the speed of light and $r_{i}$ is the range between satellite $i$ and the receiver.

By rewriting $r_{i}$ with the receiver to satellite vector, $\vec{r}_{i}$ and its unit vector, $\hat{r}_{i}$ and by using the position vectors of the satellite and receiver, $\vec{S}_{i}$ and $\vec{R}$ respectively, the pseudorange is as follows:

$$
P_{i}=c b_{r x}+\hat{r}_{i} \cdot \vec{S}_{i}-\hat{r}_{i} \cdot \vec{R}
$$

The geometry matrix of the satellite constellation, $G$, is as follows:

$$
G=\left(\begin{array}{cccc}
u_{r_{1}}^{x} & u_{r_{1}}^{y} & u_{r_{n}}^{z} & -1 \\
\vdots & \vdots & \vdots & \vdots \\
u_{r_{n}}^{x} & u_{r_{n}}^{y} & u_{r_{n}}^{z} & -1
\end{array}\right)
$$

where $u_{r_{i}}^{x}, u_{r_{i}}^{y}$ and $u_{r_{i}}^{z}$ are components of $\hat{r}_{i}$ and $\mathrm{n}$ represents the satellite number, for $\mathrm{n} \geq 4$.

Now by re-arranging eq. 2.15 :

$$
\hat{r_{i}} \cdot \vec{R}-c b_{r x}=\hat{r}_{i} \cdot \vec{S}_{i}-P_{i}
$$

and by using the same notation, $G$ becomes:

$$
G\left(\begin{array}{l}
x \\
y \\
z \\
c b_{r x}
\end{array}\right)=\left(\begin{array}{c}
\hat{r}_{1} \cdot \vec{S}_{1}-P_{1} \\
\vdots \\
\hat{r}_{n} \cdot \vec{S}_{n}-P_{n}
\end{array}\right)
$$

The solution to eq. 2.18 is then found by taking the generalised inverse of $G,\left(G^{T} G\right)^{-1} G^{T}$ as follows:

$$
\left(\begin{array}{l}
x \\
y \\
z \\
c b_{r x}
\end{array}\right) \simeq\left(G^{T} G\right)^{-1} G^{T}\left(\begin{array}{c}
\hat{r}_{1} \cdot \vec{S}_{1}-P_{1} \\
\vdots \\
\hat{r}_{n} \cdot \vec{S}_{n}-P_{n}
\end{array}\right)
$$

The receiver coordinates are initially unknowns, yet are needed to find a solution. Therefore, iteration is necessary and a guess, such as the centre of the Earth, must be given at the start. Four or five iterations will yield a solution.

\subsection{Common Pseudorange Corrections}

The following corrections, from Parkinson and Spilker (1996), are applied to each of the timing solutions to account for the Sagnac effect, the tropospheric delay and the satellite clock bias.

\section{- The Sagnac bias, $T_{\text {sagn }}$}

The Sagnac bias, $T_{\text {sagn }}$ is calculated to account for the Earth's rotation during the propagation time of the GPS signal. The receiver coordinates must be known (or approximated) beforehand.

$$
T_{\text {sagn }} \simeq c^{-1} \vec{\Omega} \cdot \vec{S} \times \vec{R}
$$

Where $c, \vec{\Omega}, \vec{S}$ and $\vec{R}$ represent the speed of light, the rotation vector of the Earth, the satellite position vector and the receiver position vector respectively.

\section{- Tropospheric delay, $T_{\text {trop }}$}

The error imposed upon the GPS signal as it propagates through the troposphere, is approximated and corrected for as follows:

$$
T_{\text {trop }} \simeq T_{\pi / 2,0} \frac{1.021 \times e^{-0.133 \times 10^{-3} h}}{\sin \phi+0.0121}
$$

The azimuth delay at sea level, the receiver altitude and the elevation angle of the satellite are represented as follows; $T_{\pi / 2,0} \approx 2.44 m, h$ and $\phi$ respectively.

\section{- Satellite clock bias, $b_{s v}$}

The satellite clock bias $b_{s v}$ accounts for the satellite specific error $\Delta_{s}^{\prime} t$ and the satellite relativistic effects $\Delta_{s}^{r} t$ and is corrected for according to Kouba and Heroux (2001) as follows:

$$
c \Delta_{s}^{r} t=2 S \dot{S} c^{-1} \Rightarrow c b_{s v}=c \Delta_{s}^{\prime} t+2 S \dot{S} c^{-1}
$$


where $S$ and $\dot{S}$ represent the distance between the satellite and center of the reference frame and the rate of change of this distance respectively.

The product of these fundamental and common corrections results in a set of partially corrected pseudorange equations for each satelliteto-receiver ray path, which lays the foundation for each GPS timing solution. Only the method by which the ionospheric delay is to be accounted for remains.

\subsection{Ionospheric corrections and the five timing solutions}

The method of ionospheric compensation employed by each of the five timing solutions is now described below.

\section{- Single-frequency solution and no ionospheric} correction

A single-frequency solution is obtained by extracting the $P 1$ code from the RINEX file. This represents the first GPS timing solution and does not include any correction for the ionosphere (only the common pseudorange corrections) and illustrates the sheer magnitude of the ionospheric delay.

\section{- Single-frequency solution and the Klobuchar model}

The second technique incorporates the Klobuchar model for ionospheric correction. This is a global model of the ionosphere and is represented by 8 coefficients, which are broadcast along with the GPS navigation message. Standard single-frequency GPS receivers use this model to correct for the ionosphere in realtime, via a receiver based algorithm. This solution portrays the current accuracy of such receivers. The data are recorded within the RINEX navigation file and are obtained from the IGS. Typically, a minimum of 50\% RMS correction is obtained. Please refer to Klobuchar (1987) for more information.

- Single-frequency solution with IRI model

The IRI 2001 model (Bilitza, 2001) offers a global, 3D description of the electron density, given the solar activity and a particular date/time. This model is not available for realtime applications.

Forward integration along slant paths of the model provide an estimate of the ionospheric delay imposed upon an $L 1$ signal, using eq. 2.23 below:

$$
T_{\text {iono }}=\frac{40.3}{c f^{2}} \int N d l
$$

where $c$ is the speed of light, $f$ is the frequency of the GPS signal $(L 1,1575.42 \mathrm{MHz}), N$ is the electron concentration, provided by the model and $d l$ is the ray-path to voxel intersection.

\section{- Single-frequency solution with MIDAS}

MIDAS, together with the pure single-frequency solution, represents a new approach to GPS timing solutions. MIDAS was first presented by Mitchell and Spencer (2003) and has since been upgraded (Spencer and Mitchell, 2007). Data is input from the IGS/EPN network of dual-frequency receivers, based across Europe, in order to produce 4D real-time ionospheric maps.

The ionospheric (group) delay may be found as follows:

$$
T_{\text {iono }}=\frac{40.3}{c f^{2}} \int N d l
$$

where $\int N d l$ is the TEC in electrons per squared metre. The TEC is given by the line integral of the electron density between the satellite-to-receiver ray-path, which is provided by MIDAS.

\section{- Dual-frequency solution}

Lastly, the dual-frequency solution is used to act as a benchmark. Two GPS signals with differing frequencies are used to measure the ionospheric delay, thus this method represents what is widely considered as the best ionospheric compensation technique, provided that accurate DCB data are obtained.

The ionosphere-free pseudorange, called $P_{0}$ here, is used to calculate the dual-frequency solution. $P_{0}$ is computed from four observables, $P_{1}$ and $P_{2}$, which represent the pseudoranges 
obtained from the precise $P$-code signal and $L_{1}$ and $L_{2}$, which are the recorded carrier phases of the signal, in terms of distance:

$$
\begin{aligned}
& P_{1}=P_{0}+T_{\text {iono }} f_{1}^{-2}+\varepsilon_{1} \\
& P_{2}=P_{0}+T_{\text {iono }} f_{2}^{-2}+\varepsilon_{2} \\
& L_{1}=P_{0}-T_{\text {iono }} f_{1}^{-2}+n_{1} \lambda_{1} \\
& L_{2}=P_{0}-T_{\text {iono }} f_{2}^{-2}+n_{2} \lambda_{2}
\end{aligned}
$$

where, $\varepsilon_{1}$ and $\varepsilon_{2}$ represent the noise, $n$ represents the integer ambiguity and $\lambda$ represents the carrier wavelength. Thus the following expressions for $T_{\text {iono }}$ may be obtained from dual-frequency data:

$$
\begin{aligned}
& \frac{P_{1}-P_{2}}{f_{1}^{2}-f_{2}^{2}}=T_{\text {iono }}+\frac{\varepsilon_{1}-\varepsilon_{2}}{f_{1}^{2}-f_{2}^{2}} \\
& \frac{L_{1}-L_{2}}{f_{1}^{2}-f_{2}^{2}}=-T_{\text {iono }}+\frac{n_{1} \lambda_{1}-n_{2} \lambda_{2}}{f_{1}^{2}-f_{2}^{2}}
\end{aligned}
$$

The first expression illustrates the ionospheric delay plus a noise term, whilst the second includes the integer ambiguity term, along with $T_{i o n o}$. Whilst a given satellite is visible, the ambiguity does not change. However, large and sudden changes can occur due to cycle slips, but these are detectable. $T_{\text {iono }}$ is hence found by fitting eq. 2.27 into eq. 2.26 to yield:

$$
\begin{aligned}
& P_{1}-T_{\text {iono }} f_{1}^{-2}=P_{0}+\varepsilon \\
& L_{1}+T_{\text {iono }} f_{1}^{-2}=P_{0}+n_{1} \lambda_{1}
\end{aligned}
$$

Fitting eq. 2.29 into eq. 2.28 then gives $P_{0}$. This forms part of the dual-frequency timing solution.

\section{Results}

Figures 2 to 5 inclusive show the diurnal variation in RCB for geomagnetically quiet and disturbed days selected from 2002, for GOPE and VILL. The minimum and maximum values for the $\mathrm{Kp}$ indices measured on each day are quoted in each corresponding figure caption. Kp values between 0-4 and 5-9 represent geo- magnetically quiet and stormy days respectively. Each figure consists of two plots, (a) and (b), which represent the fixed and mobile receiver techniques respectively. These figures illustrate the performance of MIDAS in comparison to other GPS timing solutions and in increasing order, the general accuracy is: no correction, Klobuchar, IRI 2001, MIDAS and dual-frequency.

Figures 2 to 5 inclusive show that the uncorrected solution gives the worst results because it always exhibits a significant peak around midday (as expected due to the Sun's ionizing radiation). During the night-time this solution generally provides a more stable and reduced RCB, but this is not so evident in fig. 5 and the other solutions always perform better. The Klobuchar solution also exhibits a diurnal peak, which is either positive or negative and represents the model's tendency to undercompensate or overcompensate, respectively, for the ionospheric delay. This solution provides a steady nighttime result and overall, a lower RCB than the solution with no ionospheric correction, but it is still highly variable. Generally, the IRI solution displays a small diurnal peak and despite its variability, it yields an improved RCB at nighttime and daytime, with respect to the Klobuchar and uncorrected solutions. MIDAS offers the most accurate and least variable single-frequency solution throughout the whole day. It eliminates the diurnal peak and is highly comparable to the benchmark dual-frequency solution. This yields a virtually constant RCB of 5 ns, largely irrespective of the time of day.

The solution characteristics described above are typical of both the quiet and stormy days. Figures $2 \mathrm{a}$ and $2 \mathrm{~b}$ exhibit a negative-looking spike at around 02:00 UT for the dual-frequency solution. This is due to losses of lock on both frequencies at the receiver and shows that the MIDAS solution performs better under these conditions, because it is not vulnerable to isolated losses of lock on the $L 2$ signal.

By comparing the results for a fixed receiver (see figs. 2a to 5a inclusive) to those for a mobile receiver (see figs. $2 b$ to $5 b$ inclusive), it is clear that the solutions become more variable and that the magnitude of the RCB generally increases. This is most evident for the following 


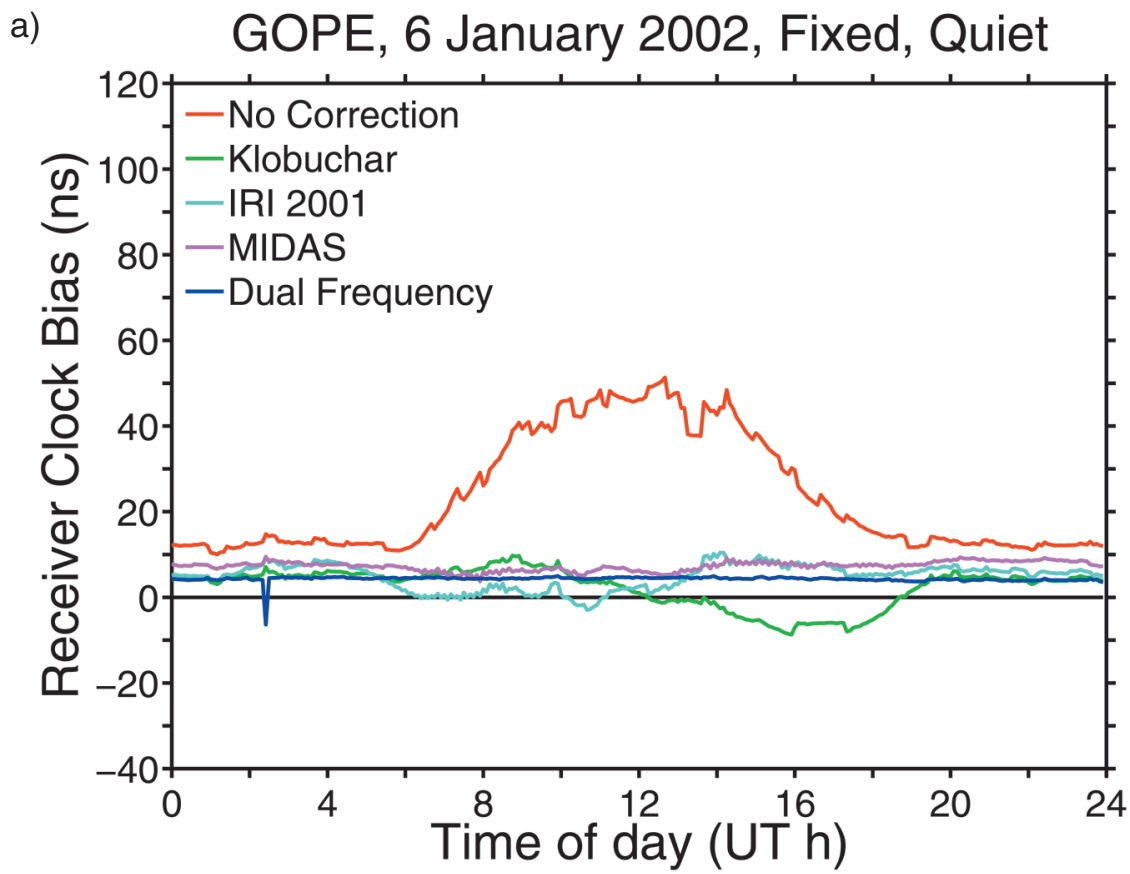

b) GOPE, 6 January 2002, Mobile, Quiet

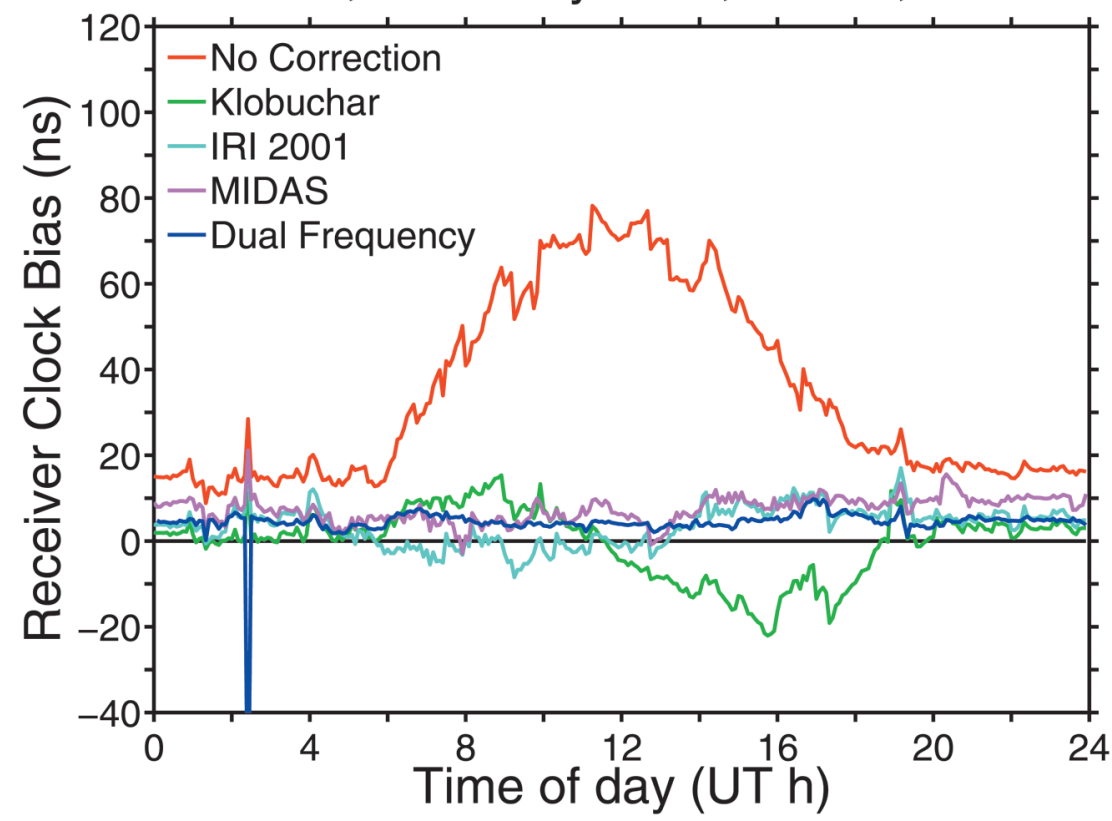

Fig. 2a,b. Receiver clock bias referenced to the receiver clock bias from CODE for GOPE 6 January 2002, for a quiet ionosphere (Kp index 0-1.3) using a fixed (a) and mobile (b) GPS receiver solution. 
a) GOPE, 21 November 2002, Fixed, Stormy

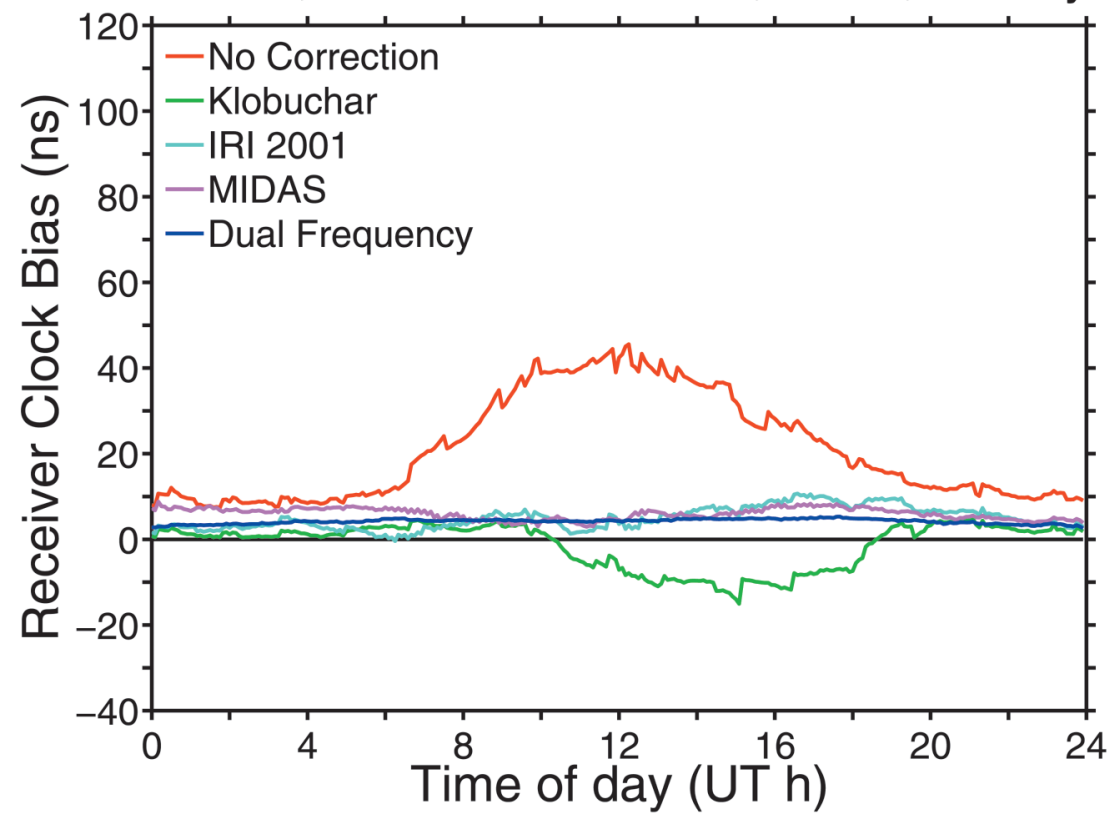

b) GOPE, 21 November 2002, Mobile, Stormy

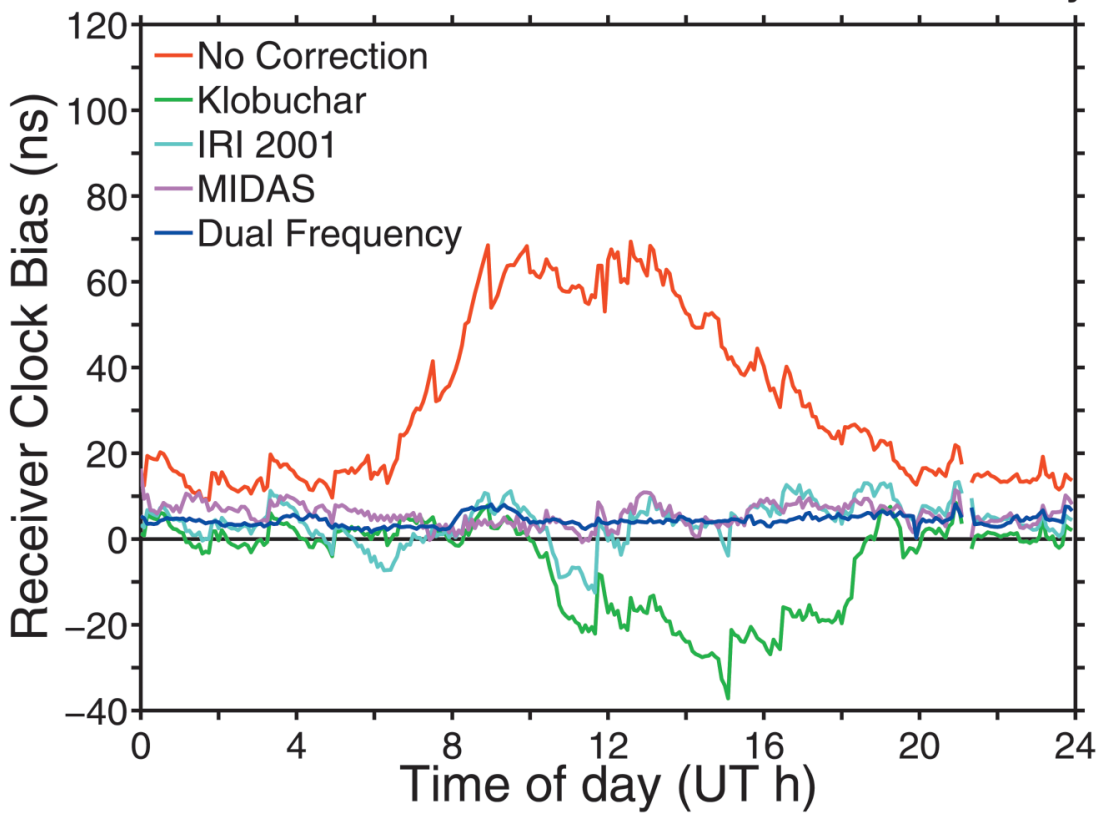

Fig. 3a,b. Receiver clock bias referenced to the receiver clock bias from CODE for GOPE 21 November 2002, for a stormy ionosphere (Kp index 4.3-6.7) using a fixed (a) and mobile (b) GPS receiver solution. 

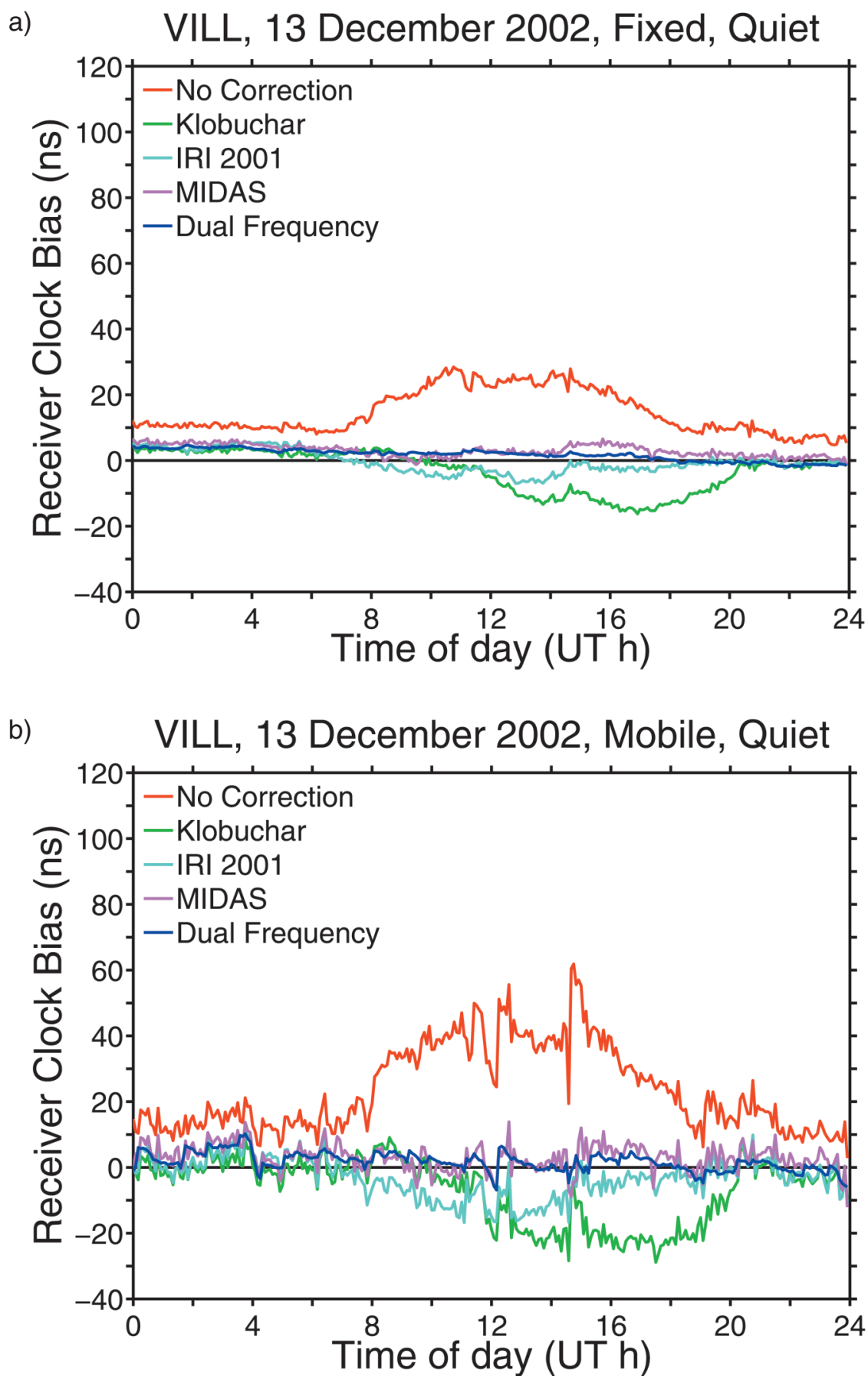

Fig. 4a,b. Receiver clock bias referenced to the receiver clock bias from CODE for VILL 13 December 2002, for a quiet ionosphere (Kp index 0.3-1.7) using a fixed (a) and mobile (b) GPS receiver solution. 
a)

VILL, 11 May 2002, Fixed, Stormy

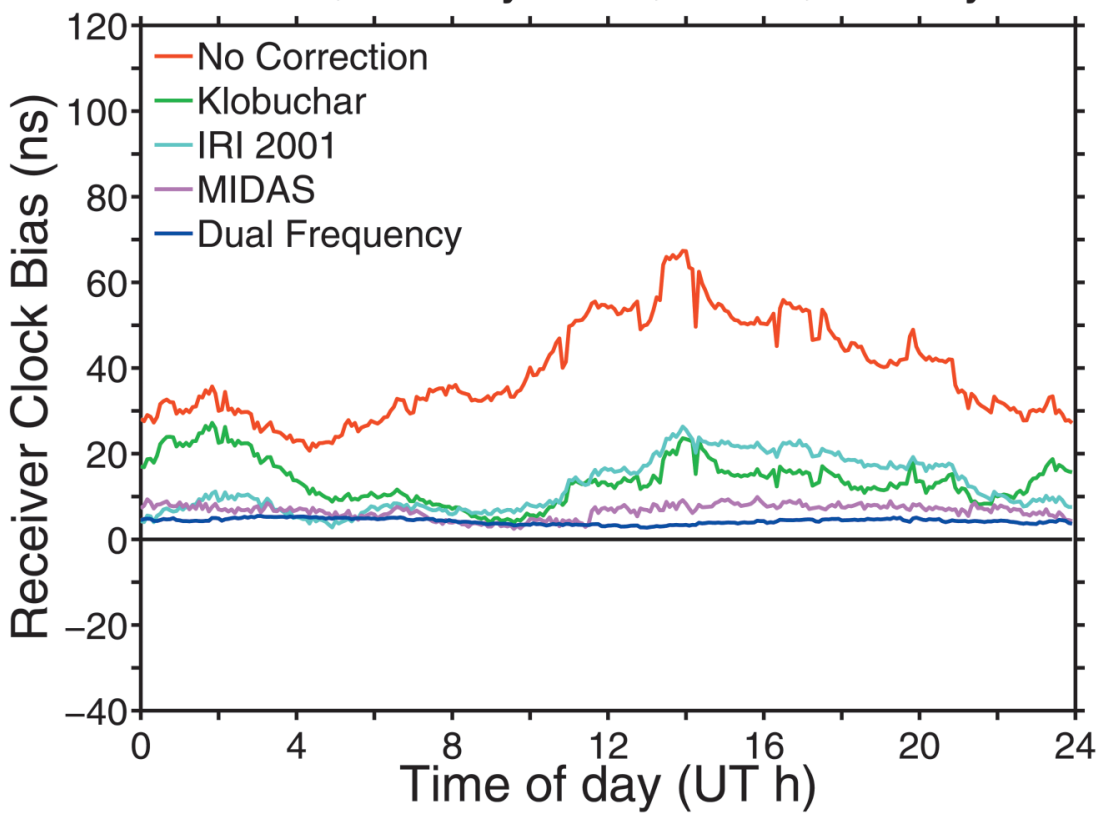

b) VILL, 11 May 2002, Mobile, Stormy

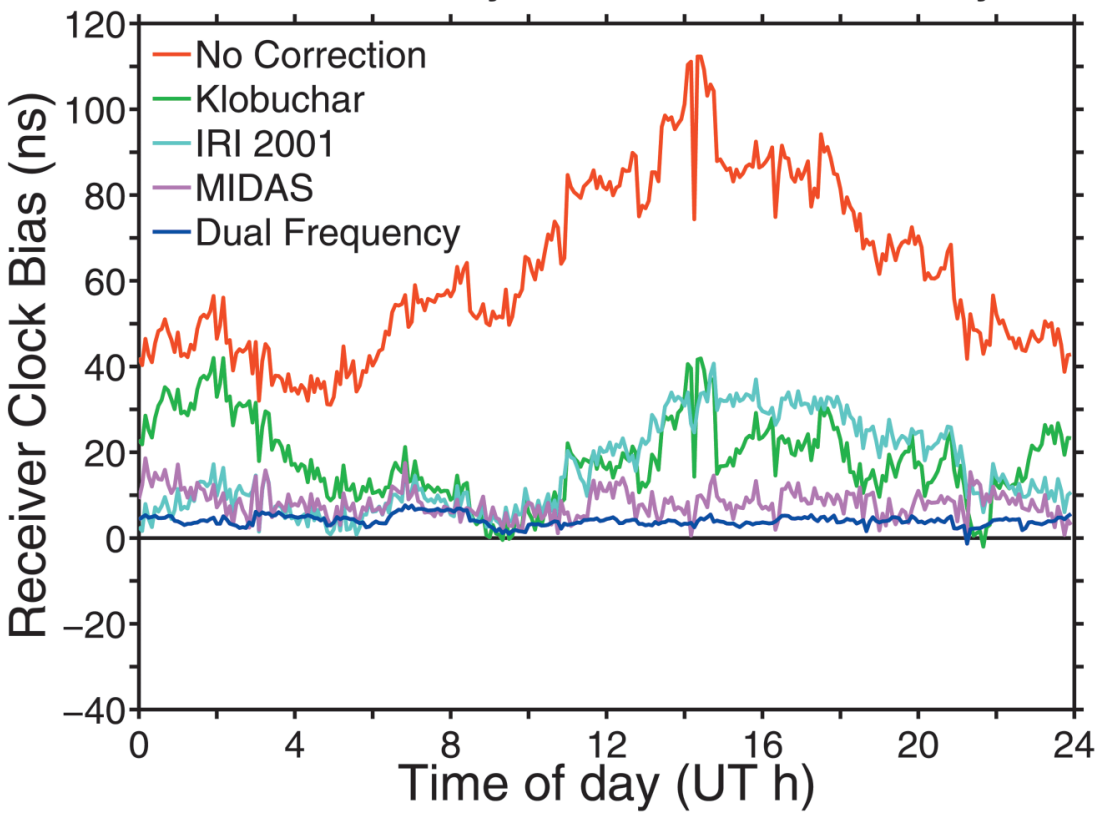

Fig. 5a,b. Receiver clock bias referenced to the receiver clock bias from CODE for VILL 11 May 2002, for a stormy ionosphere (Kp index 0.7-6.7) using a fixed (a) and mobile (b) GPS receiver solution. 


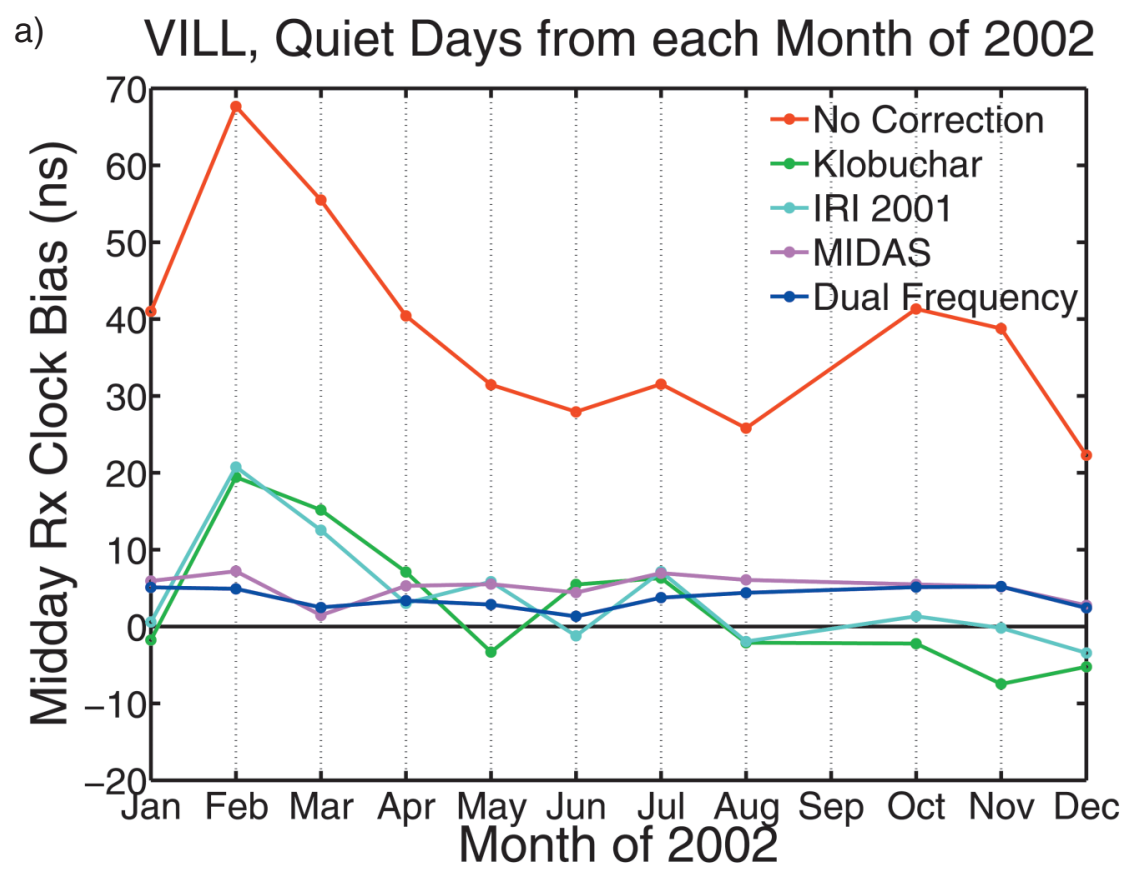

b) VILL, Stormy Days from each Month of 2002

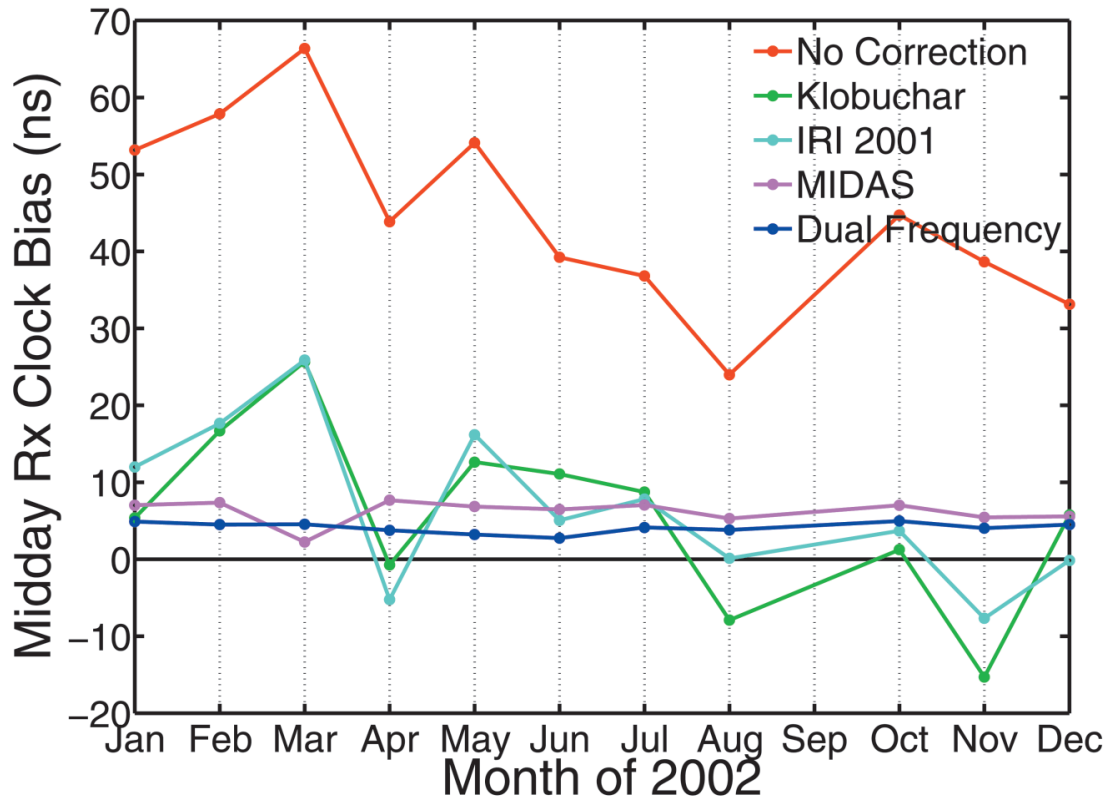

Fig. 6a,b. Midday (12:00 UT) receiver clock bias referenced to the receiver clock bias from CODE (using the fixed receiver solution) for one quiet day (a) and one stormy day (b) from each month of 2002 at VILL, excluding September due to data quality. 
a)

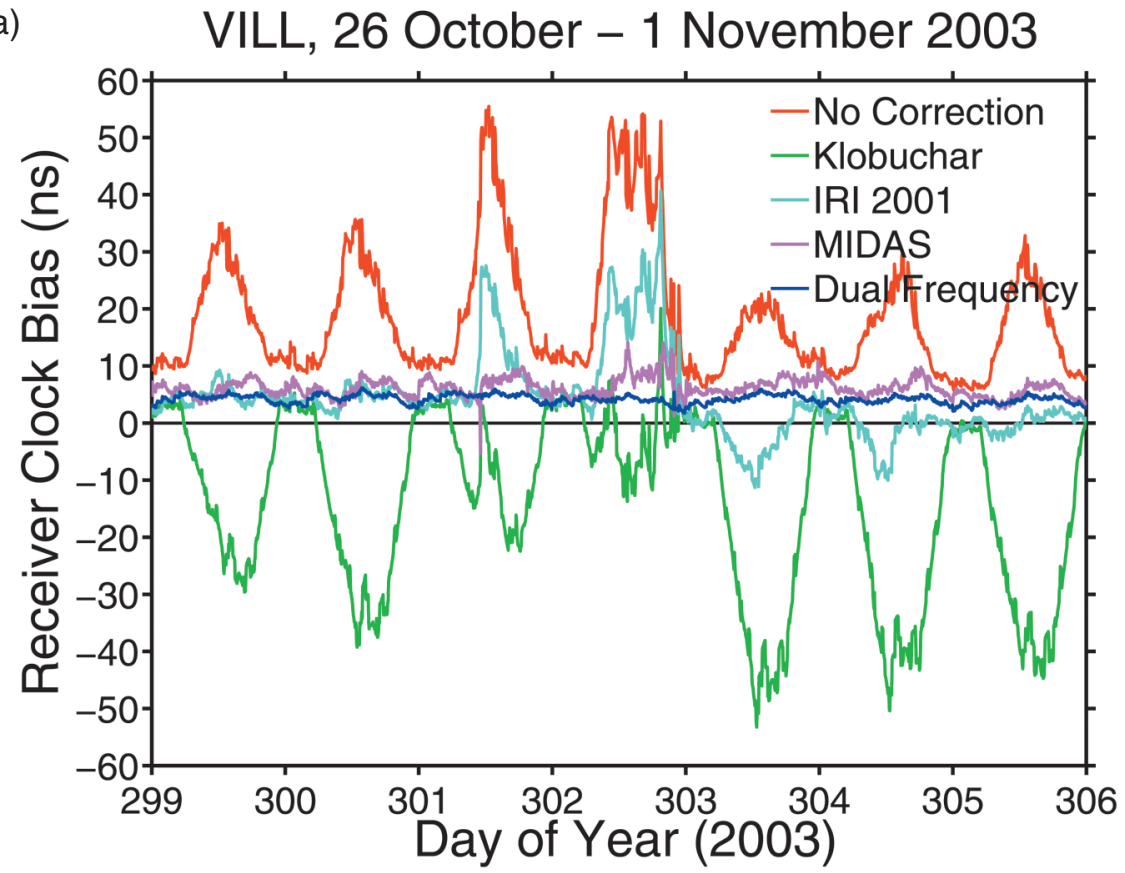

b) $\mathrm{Kp}$ Indices for 26 October - 1 November 2003

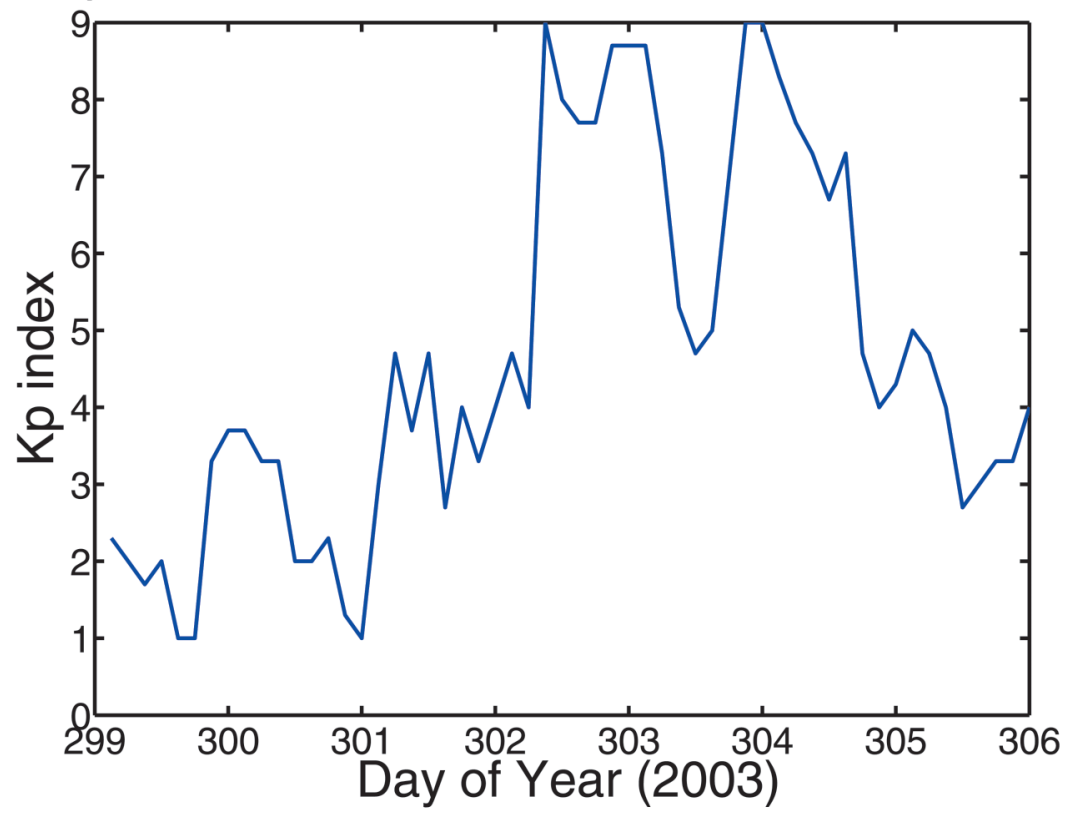

Fig. 7a,b. Receiver clock bias referenced to the receiver clock bias from CODE for VILL before, during and after the October 2003 ionospheric storm, using a fixed GPS receiver solution (a) and corresponding Kp indices (b). 
solutions: no correction, Klobuchar and IRI 2001 , which is to be expected because the receiver is constantly moving and so an approximation of its position is regularly being made, which directly affects the accuracy of the fundamental calculations and corrections described in the Method. However, the MIDAS and dual-frequency solutions continue to yield a RCB of typically $10 \mathrm{~ns}$ or less, whilst the stability of the latter solution is slightly better than that of MIDAS.

Figures $6 a$ and $6 \mathrm{~b}$ illustrate the RCB at 12:00 UT (midday) for one quiet and one stormy day, respectively, from each month in 2002, for each timing solution, at VILL. The RCB have not been included for September due to data quality. Table I summarizes the RMS midday RCB for both the quiet and stormy days selected from 2002 at VILL. These figures, together with table I, reinforce the fact that the solution accuracies, in increasing order are as follows: no correction, Klobuchar, IRI 2001, MIDAS and dual-frequency. The solution with no correction is the worst and MIDAS gives the best single-frequency GPS timing solution, which is virtually on par with the dual-frequency solution.

Figures $6 \mathrm{a}$ and $6 \mathrm{~b}$ also show a seasonal variation. The midday peak in RCB for the uncorrected solution tends to be larger in the autumn and winter months in comparison to the spring and summer months. The same is true for the Klobuchar and IRI 2001 solutions, which yield similar results to each-other. These models undercompensate at the beginning of the year (winter-spring) and compensate best during the summer, whilst overcompensating in the autumn. MIDAS and the dual-frequency solutions are very similar and the former performs slightly better in March 2002. According to the RMS values for the quiet days from table I, for each solution, MIDAS is shown to achieve an $86 \%$ and $37 \%$ RMS improvement with respect to the uncorrected and Klobuchar solutions correspondingly. The Klobuchar solution achieves its design goal of providing a minimum 50\% RMS ionospheric correction.

Figure 7a shows the variation in RCB for several GPS timing solutions over the period 26 October (day 299 of 2003) to 1 November 2003
Table I. RMS midday (12:00 UT) receiver clock bias (ns) referenced to the receiver clock bias from CODE (using the fixed receiver solution) for the quiet and stormy days selected from each month of 2002 at VILL, excluding September due to data quality.

\begin{tabular}{ccc}
\hline GPS Timing & \multicolumn{2}{c}{ RMS receiver clock bias (ns) } \\
Solution & Quiet days & Stormy days \\
\hline No correction & 40.6 & 46.0 \\
Klobuchar & 8.7 & 12.3 \\
IRI 2001 & 8.0 & 12 \\
MIDAS & 5.4 & 6.4 \\
Dual-frequency & 4.0 & 4.2 \\
\hline
\end{tabular}

(day 305 of 2003) inclusive, at VILL, using a fixed receiver solution. Two Coronal Mass Ejections (CME) were detected on days 302 and 303 (Skoug et al., 2004). This led to global and intense geomagnetic storms, as reflected by the corresponding high $\mathrm{Kp}$ indices in fig. $7 \mathrm{~b}$. VILL is in Spain and it is important to note that days 302 and 303 mark the positive and negative phases of the storm respectively, across this region. Figure $7 \mathrm{~b}$ shows that days 299 and 300 mark a depression in the ionosphere and are essentially quiet, which is reinforced by the corresponding low peak of about $35 \mathrm{~ns}$ in RCB in fig. $7 \mathrm{a}$, for the solution with no correction. In contrast, day 301 is moderately disturbed and with reference to figs. 2 to 5 inclusive, a midday peak in RCB of about 55 ns for the solution with no correction is not unusual, as seen on day 301 in fig. 7a. Days 304 and 305 show the RCB after the storm. Figure 7a shows that the Klobuchar solution always overcompensates for the ionospheric delay and would indeed make the resultant timing solution worse on days 300, 303, 304 and 305, in comparison to the solution with no ionospheric correction. This was when the ionosphere was depressed and/or with very low TEC. A prolonged and highly variable RCB peak is seen in fig. 7a on day 302 , for the curve with no correction and is due to the intensely disturbed ionosphere, which exists not only during the daytime but continues deep into the night because of the 
positive phase of the storm. The same curve for day 303 exhibits a much lower peak than normal, at about $22 \mathrm{~ns}$ and is due to the negative phase of the storm, which causes a depression in the ionosphere. Overall, the IRI model is unstable and exhibits significant midday peaks. In contrast, the MIDAS and dual-frequency solutions provide fairly stable and comparable solutions throughout the intense period.

\section{Conclusions and discussion}

A new single-frequency GPS timing solution that uses tomography to correct for the ionospheric delay has been presented and compared to various other GPS timing solutions in this paper. Results were produced using fixed and mobile based receiver techniques for two IGS stations, VILL (Spain) and GOPE (Czech Republic).

In increasing order, the general timing accuracy is: no correction, Klobuchar, IRI 2001, MIDAS and dual-frequency. It should be noted that in certain circumstances the Klobuchar solution can produce a worse result than if there were no ionospheric correction at all, as seen by the extreme ionospheric conditions presented in fig. 7a. MIDAS offers the best single-frequency solution because it is typically very stable and eliminates the large RCB seen at midday by the other single-frequency solutions. These characteristics are true for the fixed and mobile receiver solutions. The RMS RCB of the GPS timing solutions with no ionospheric correction, the Klobuchar model and the IRI model noticeably increase and become more variable under stormy conditions, as opposed to quiet conditions. This is in contrast to the MIDAS and dual-frequency solutions which continue to provide stable and accurate results under variable ionospheric conditions.

Overall MIDAS is shown to achieve accuracies comparable to those from the dual-frequency system presented here and is in some cases more robust as it is not vulnerable to $L 2$ losses of lock. For stormy days, MIDAS provides a stable GPS timing solution accurate to within 6.4 ns RMS, typically correcting $86 \%$ of the error imposed by the ionosphere (according to the solution with no correction). In comparison, the broadcast Klobuchar model exhibits a $73 \%$ reduction in the ionospheric error, but as figs. 2 to 7 inclusive show, it can be highly variable (over the course of a few minutes to a few hours) and unreliable in severe conditions.

MIDAS corrections could be freely broadcast to GPS end-users in real-time. The corrections are created using GPS data, which are available from an existing infrastructure and would be pre-processed at a central control station which would then broadcast the corrections (and/or via the internet) to the end-users in real-time, isolating them from the specialist equipment and processing. This would enable more-accurate GPS timing solutions to be obtained from standard low-cost single-frequency GPS receivers than is currently possible using the broadcast corrections, albeit with a modification enabling them to interpret the MIDAS data. Applications that rely on GPS for timing purposes are now more widespread than during the last solar maximum and so it might be prudent to employ a system that provides greater accuracy than standard single-frequency receivers. Solar maxima give rise to increases in ionospheric storms and radiation, which can seriously degrade the GPS solution if left uncompensated. Telecommunications (wired/wireless), power transmission and cosmic ray detectors are just three applications that require accurate timing information and/or synchronisation. More specifically, timing data is used in telecommunication systems to maintain carrier frequencies and in banking networks towards the synchronisation of data encryption equipment (Dana and Penrod, 1990). The system proposed here can be used to synchronise two (or more) free running devices, anywhere in the world, using GPS, to within $10 \mathrm{~ns}$. It could help to reduce the risks of poor synchronisation, which can seriously degrade network performance and can lead to loss of revenue, during highly variable ionospheric conditions.

\section{Acknowledgments}

We would like to express our gratitude to the UK Engineering and Physical Sciences Re- 
search Council (EPSRC) for funding this project. We are grateful to the IGS and EPN for the GPS data, the UKSSDC for the Kp data and we are also thankful for the use of the IRI model.

\section{REFERENCES}

Allain, D.J. and C.N. Mitchell (2009): Ionospheric delay corrections for single-frequency GPS receivers over Europe using tomographic mapping, GPS Solutions, 13 (2), 141-151, doi:10.1007/s10291-008-0107-y.

Beutler, G., M. Rothacher, S. Schaer, T.A. Springer, J. KouBA and R.E. NeILAN (1999): The International GPS Service (IGS): An interdisciplinary service in support of Earth sciences, Adv Space Res., 23 (4), 631-653.

BILITZA, D. (2001): IRI 2000, Radio Science, 36 (2), 261-276.

BRUYNINX, C. (2004): The EUREF Permanent Network: a multi-disciplinary network serving surveyors as well as scientists, GeoInformatics, 7, 32-35.

Bust, G.S. and C.N. Mitchell (2008): History, current state, and future directions of ionospheric imaging, Rev. of Geophys., 46 (1), doi:10.1029/2006RG000212.

DANA, P.H. and B.M. PENROD (1990): The role of GPS in precise time and frequency dissemination, GPS World, 38-43.

DAVIES, K. (1990): Ionospheric Radio, IEE Electromagnetic Waves, (Peter Peregrinus Ltd., London, UK).

Dow, J.M., R.E. NeILAN and G. GENDT (2005): The international GPS service: celebrating the 10th anniversary and looking to the next decade, Adv. Space Res., 36 (3), 320-326.

GuRTNER, W. (2002): RINEX: The Receiver Independent Exchange Format Version 2.10, Bull. Am. Meteorol. Soc., 77, 1-18.

KLOBUCHAR, J.A. (1987): Ionospheric time-delay algorithm for single-frequency GPS users, IEEE Trans Aerosp Electron Syst., 23 (3), 325-331.

KouBA, J. and P. Heroux (2001): GPS precise point position- ing using IGS orbit products, GPS Solutions, 5 (2), 12-28. KOUBA, J. and T. SPRINGER (2001): New IGS Station and Satellite Clock Combination, GPS Solutions, 4 (4), 31-36.

LANGLEY, R.B. (1997): The GPS Error Budget, GPS World, 8 (3), 51-56.

LeWANDOWSKI, W. and J. AzoubIB (2000): Time transfer and TAI. Frequency Control Symposium and Exhibition, 2000, Proceedings of the 2000 IEEE/EIA International, 586-597.

MegGs, R.W. and C.N. Mitchell (2006): A study into the errors in vertical total electron content mapping using GPS data, Radio Science, 41 (1).

Mitchell, C.N. and P.S.J. SPENCER (2003): A three-dimensional time-dependent algorithm for ionospheric imaging using GPS, Ann. Geophysics, 46 (4), 687-696.

PARKINSON, B.W. and J.J. SPILKER (1996): Global positioning system: theory and applications, vol. 1, AIAA.

RAY, J. and K. SENIOR (2003): IGS/BIPM pilot project: GPS carrier phase for time/frequency transfer and timescale formation, Metrol., 40 (4), 205-205.

Schmid, R., P. Steigenberger, G. Gendt, M. Ge and M. ROTHACHER (2007): Generation of a consistent absolute phase-center correction model for GPS receiver and satellite antennas, J. Geod., 81 (12), 781-798.

Skoug, R.M., J.T. Gosling, J.T. Steinberg, D.J. MccoMAS, C.W. SMith, N.F. Ness, Q. Hu and L.F. BuRLaGa (2004): Extremely high speed solar wind: 29-30 October 2003, J. Geophys. Res., 109 (A9), A09102.

Smith, D.A., E.A. Araujo-Pradere, C.F. Minter and T.J. FULLER-ROwELl (2008): A comprehensive evaluation of the errors inherent in the use of a two-dimensional shell for modeling the ionosphere, Radio Science, 43, RS6008, doi:10.1029/2007RS003769.

SPENCER, P.S.J. and C.N. MitCHELL (2007): Imaging of fast moving electron-density structures in the polar cap, Ann. Geophysics, 50 (3), 427-434.

(received, June 1, 2009; accepted, July 7, 2009) 